ARTICLE

Received 29 Oct 2013 | Accepted 31 Dec 2013 | Published 23 Jan 2014 DOl: 10.1038/ncomms4184

\title{
Female monopolization mediates the relationship between pre- and postcopulatory sexual traits
}

\author{
Stefan Lüpold', Joseph L. Tomkins², Leigh W. Simmons² \& John L. Fitzpatrick ${ }^{2,3}$
}

Theory predicts a trade-off between investments in precopulatory (ornaments and armaments) and postcopulatory (testes and ejaculates) sexual traits due to the costs associated with their growth and maintenance within the finite energy resources available. Empirical studies, however, have revealed considerable inconsistency in the strength and direction of relationships among these sexual traits. Ambiguity may result from variance in the marginal benefits gained by increasing investments in either pre- or postcopulatory sexual traits. Here, in a broad comparative study, we test the prediction that the relationship between pre- and postcopulatory sexual traits differs among taxa relative to the importance of male-male contest competition within them. We find that covariance between pre- and postcopulatory sexual traits gradually shifts from strongly positive to strongly negative with increasing male-male contest competition. Thus, our findings reveal a potentially unifying explanation for the oftentimes inconsistent relationships in the strength and direction of covariance among sexual traits.

\footnotetext{
${ }^{1}$ Department of Biology, Life Sciences Complex, 107 College Place, Syracuse University, Syracuse, New York 13244-1270, USA. ${ }^{2}$ Centre for Evolutionary Biology, School of Animal Biology (M092), University of Western Australia, Crawley, Western Australia 6009, Australia. ${ }^{3}$ Computational and Evolutionary Biology, Faculty of Life Sciences, University of Manchester, Manchester M13 9PT, UK. Correspondence and requests for materials should be addressed to S.L. (email: sluepold@gmail.com).
} 
P recopulatory sexual selection is one of the primary forces underlying the evolution of extravagant phenotypic structures, including weapons used in male-male combat over access to females and/or male ornaments to attract females ${ }^{1,2}$. The bearer's success in acquiring matings is typically influenced by the size or elaboration of these sexually selected traits ${ }^{2}$. However, since females often mate with multiple males ${ }^{3}$, sexual selection can continue after mating, with sperm from different males competing for fertilization of a female's ova ${ }^{4}$ and/or females biasing the chances of fertilization among competing ejaculates ${ }^{5,6}$. Consequently, a male's overall fitness is determined by his competitiveness during both pre- and postcopulatory selective episodes ${ }^{7,8}$. Yet, while these episodes have separately attracted much attention in the study of sexual traits, very little is known about their interaction in explaining patterns of trait evolution.

Producing and maintaining weapons used in precopulatory contest competition and ejaculate traits important in postcopulatory sperm competition can be energetically demanding ${ }^{9-12}$. Consequently, sperm competition models assume that energy allocated to mate acquisition limits investment in ejaculate production, leading to the prediction that investment in testes should trade off against the investment in weaponry used in male-male contest over access to mates ${ }^{13}$. Nevertheless, generating predictions for the direction and strength of the presumed covariance between pre- and postcopulatory traits can be complex. For example, the expression of trade-offs between pre- and postcopulatory traits is likely to depend on the marginal benefits gained by increasing investment in either pre- or postcopulatory sexual traits. To examine how variance in allocation strategies differentially influences the evolution of sexual traits, Parker et al. ${ }^{13}$ described the mate-competition loading, $a$, which refers to the marginal reproductive gains associated with increasing investment in weaponry. Specifically, reproductive gains associated with increased investment in precopulatory weaponry outweigh the added costs of expenditure on weaponry when $a>1$ (that is, in cases of malemale contest competition rather than scramble competition). Parker et al. ${ }^{13}$ then predicted that investment in ejaculates should decrease with any increase in either $a$ or the number of males competing for a given mating. Because of the covariance between $a$ and the number of competing males, pre- and postcopulatory reproductive investments would be expected to trade off when $a>1$ (contest competition) but not when $a \leq 1$ (scramble competition). Consequently, the relationship between investment in pre- and postcopulatory traits may vary among taxa depending on the relative importance of male-male contest competition for mate monopolization.

To date, only few studies have examined the relationship between investments in pre- and postcopulatory sexual traits. Intraspecifically, male investment in weaponry used in male-male competition (for example, horns, large male size relative to females, or grasping forearms) appears to be negatively associated with testes size or ejaculate size in most species examined (for example, dung beetles Onthophagus nigriventris ${ }^{14}$, dung flies Sepsis punctum ${ }^{15}$, myobatrachid frogs Crinia georgiana ${ }^{13}$ and freshwater crayfish Austropotamobius italicus $\left.{ }^{16}\right)$. However, antler size covaries positively with relative testes size and sperm quality in the red deer Cervus elaphus ${ }^{17}$, and relative testes size does not differ between male tree weta Hemideina crassidens exhibiting small and large mandibular weaponry, respectively ${ }^{18}$. Similar inconsistencies are found at the macroevolutionary scale. For example, negative covariance between investment in precopulatory weaponry (in this case the degree of male body size exceeding female size) and either testicular or genital size have been documented across a range of acanthocephalan parasitic worms ${ }^{19}$ and pinnipeds (that is, seals, sea lions and walrus $)^{20}$, but no significant relationships were found across onthophagine dung beetles (Coleoptera: Scarabaeidae ${ }^{14}$ ) or a wide range of mammals ${ }^{21}$. Finally, across bushcrickets (Orthoptera: Tettigoniidae), the size of the sperm-containing ampulla of spermatophores, and thus ejaculate size, increases with the degree of sexual size dimorphism ${ }^{22}$. The current ambiguity in our understanding of the relationship between pre- and postcopulatory traits represents a major impediment to our understanding of reproductive trait evolution.

Comparing the direction of macroevolutionary trends among different taxa can reveal important information on how variance in allocation strategies differentially influences the evolution of sexual traits. However, such a systematic comparison, including an empirical test of Parker et al.' ${ }^{13}$ prediction, is yet to be done. Here, we studied interspecific associations between pre- and postcopulatory sexual traits in both vertebrate and invertebrate taxa. We then combined our findings with previously published data from additional taxa to test Parker et al.'s ${ }^{13}$ prediction that intra-taxonomic differences in the occurrence of female monopolization influence the covariance between sexual traits important in pre- and postcopulatory sexual selection. Specifically, we assumed that in taxa where males are rarely able to monopolize females, male-male competition before and after mating would be equally important, such that selection would favour both pre- and postcopulatory sexual traits and generate positive covariation between them. By contrast, if female monopolization is very common and thus the mate-competition loading $a$ high within a taxon, investments in precopulatory male-male competition should be more important. Consequently, investments in postcopulatory sexual traits would decrease relative to those in precopulatory traits either through differential allocation or because intense female monopolization would increasingly limit the opportunities for sperm competition (and thus the value of investing in postcopulatory traits). Our overall prediction based on Parker et al.'s ${ }^{13}$ models therefore was that an increasing value of $a$ (or the importance of female monopolization within a taxon) would gradually shift the strength and direction of intra-taxonomic covariation between pre- and postcopulatory sexual traits from strongly positive to strongly negative.

In accordance with Parker et al.'s ${ }^{13}$ predictions, we find that the relationship between pre- and postcopulatory sexual traits gradually shifts from strongly positive to strongly negative with increasing male-male contest competition. This dependence on the prevalence of male-male contest competition provides a potentially unifying explanation for why the strength and direction of covariance among sexual traits often varies among studies.

\section{Results}

Relationships between pre- and postcopulatory sexual traits. We collected data on pre- and postcopulatory sexual traits from the literature for seven taxa, including mammals (primates, ungulates), birds (pheasants and allies), fish (minnows), insects (stalk-eyed flies, dung beetles) and schistosomes (blood-flukes). In all these taxa, sexually selected traits important in both selective episodes are well characterized (Supplementary Note 1). Within each taxon, we examined the relationship between the expression of sexually dimorphic traits under precopulatory sexual selection (for example, sexual size dimorphism, the length and dimorphism of canines, size of horns and antlers, tarsal spur length or the span of eye stalks) with interspecific variation in relative testes size (combined testes mass or mean testis length; testes number in schistosomes), a commonly used proxy for the 
strength of postcopulatory sexual selection ${ }^{23-26}$. All sexually selected traits are described in the Methods section; for complete data sets and phylogenies see Supplementary Tables 1-6, Supplementary Figs 1-6 and ref. 27 (for minnows).

We first focused our analysis on the relationship between sexual size dimorphism (SSD), a proxy for the strength of precopulatory sexual selection ${ }^{28-30}$, and testes size as a proxy for postcopulatory sexual selection (see above), using phylogenetic multiple regressions controlling for body size (mass or length; Supplementary Note 1). Importantly, two previous studies demonstrated that among species of acanthocephalan parasitic worms (Acanthocephala) ${ }^{19}$ and pinnipeds ${ }^{20}$, the levels of SSD are inversely related to investment in testicular tissue, showing that the negative covariance between pre- and postcopulatory investment modelled by Parker et al. ${ }^{13}$ can be expressed in these traits. However, we found either no, or positive, covariation between precopulatory sexual traits and investment in testicular tissue in our interspecific comparisons, and no evidence of negative relationships between these traits. For example, testes size increased with SSD in minnows and pheasants and allies, and it tended to increase in schistosomes, albeit not significantly so (Table 1). In contrast, testes size was not correlated with SSD in any other taxon examined (including primates, ungulates and stalk-eyed flies; Table 1 and Supplementary Table 7).

Next, we examined the relationship between investment in testes size and weapons (canines, horns, antlers, spurs, eye span) known to influence the outcome of precopulatory male-male contests. These analyses revealed that, after controlling for body size, testes size did not covary significantly with canine length among primates, horn and antler size among ungulates, horn length among dung beetles, tarsal spur length among pheasants and their allies, or eye span among stalk-eyed flies (Table 2). Similarly, in taxa where both males and females express weapons, testes size was not correlated with sexual dimorphism in weapon size (Supplementary Table 8 ).

As Tables 1 and 2 indicate, the phylogenetic scaling parameter $\lambda$ varied considerably among taxa, with values being comparable between the analyses using SSD and weapon size, respectively. Whereas the reported relationships appeared to be largely independent of the underlying phylogeny in the ungulates, a very strong phylogenetic signal was found in other taxa, such as the pheasants and allies or the schistosomes (Tables 1 and 2). The $\lambda$ value for the stalk-eyed flies was very small in both cases $(<0.0001)$, but the models were also not significantly different from models with $\lambda$ set to 1 , which is likely to be due to the very small sample size. Despite this variation in $\lambda$, however, there was no inter-taxonomic relationship between $\lambda$ and the intrataxonomic sample sizes (that is, numbers of species), percentages of female monopolization or weighted $Z_{r}$ values (Pearson's product moment correlations, all $P>0.26$ ). Consequently, our limited data do not currently suggest a link between macroevolutionary patterns and the phylogenetic history of the taxa,

Table 1 | Body-size controlled relationships between testes size and sexual size dimorphism.

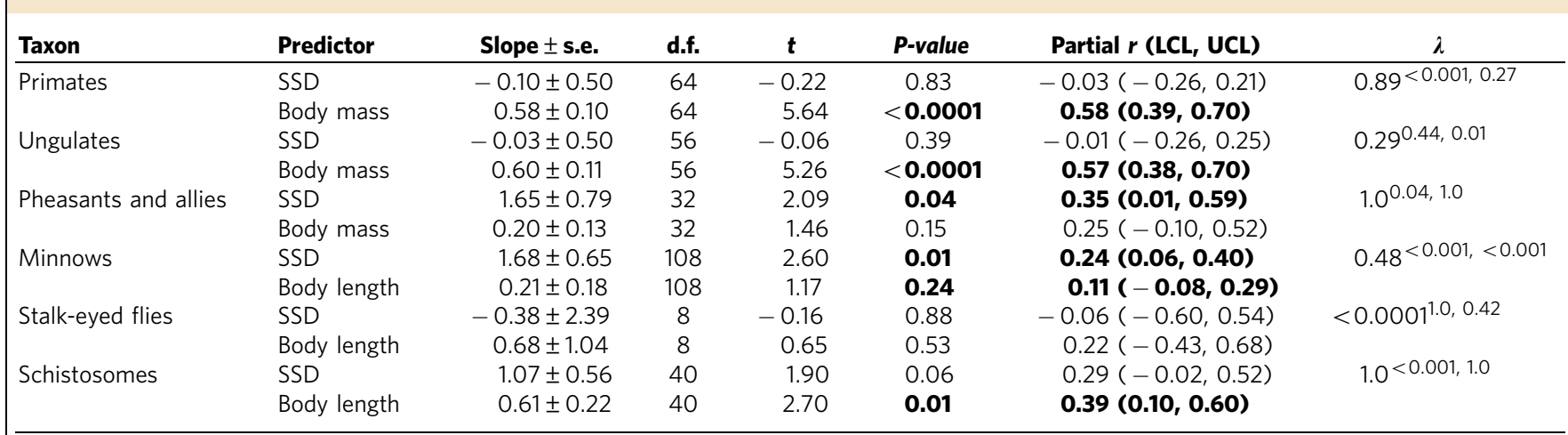

SSD, sexual size dimorphism. All analyses are controlled for phylogeny and have testes size (or testes number for schistosomes) as the dependent variable (all variables log-transformed). The partial correlation coefficients $r$ are presented with the non-central $95 \%$ confidence intervals (LCL, lower confidence limit: $U C L$, upper confidence limit), with d. f degrees of freedom; $t$ - - statistics of the phylogenetic generalized least-squared regressions and $\lambda$, phylogenetic scaling parameters (superscripts following $\lambda$ denote $P$-values of likelihood ratio tests against models with $\lambda=0$ and $\lambda=1$, phylogenetic generalized least-squared regressions and $\lambda$, phylogen
respectively). Significant relationships are indicated in bold text.

Table 2 | Body-size controlled relationships between testes size and the size of male weaponry.

\begin{tabular}{|c|c|c|c|c|c|c|c|}
\hline Taxon & Predictor & Slope \pm s.e. & d.f. & $t$ & P-value & Partial r (LCL, UCL) & $\lambda$ \\
\hline \multirow[t]{2}{*}{ Primates } & Canine height & $0.07 \pm 0.46$ & 51 & 0.15 & 0.88 & $0.02(-0.25,0.28)$ & $0.89<0.001,0.11$ \\
\hline & Body mass & $0.68 \pm 0.20$ & 51 & 3.47 & 0.001 & $0.44(0.19,0.61)$ & \\
\hline Ungulates & Horn/antler length & $0.07 \pm 0.23$ & 61 & 0.03 & 0.98 & $0.003(-0.24,0.25)$ & $0.52^{0.10,0.04}$ \\
\hline \multirow[t]{2}{*}{ Dung beetles } & Horn length & $0.10 \pm 0.09$ & 19 & 1.15 & 0.26 & $0.26(-0.20,0.60)$ & $<0.0001^{1.0,0.006}$ \\
\hline & Body mass & $0.81 \pm 0.07$ & 19 & 11.90 & $<0.0001$ & $0.94(-0.87,0.97)$ & \\
\hline Pheasants and allies & Spur length & $0.02 \pm 0.21$ & 37 & 0.10 & 0.92 & $0.02(-0.34,0.37)$ & $0.9^{0.01,0.38}$ \\
\hline Stalk-eyed flies & Body length & $-0.02 \pm 1.45$ & 8 & -0.02 & 0.99 & $-0.01(-0.60,0.60)$ & \\
\hline
\end{tabular}

All analyses are controlled for phylogeny and have testes size as the dependent variable (all variables log-transformed). The partial correlation coefficients $r$ are presented with the non-central $95 \%$ confidence intervals (LCL, lower confidence limit; UCL, upper confidence limit), with d.f., degrees of freedom, $t$, $t$-statistics of the phylogenetic generalized least-squared regressions and $\lambda=$ phylogenetic scaling parameters (superscripts following $\lambda$ denote $P$-values of likelihood ratio tests against models with $\lambda,=0$ and $\lambda=1$, respectively). Significant relationships are indicated in bold text. 
and it is beyond the scope of our study to try to understand the role of the phylogenetic signal in the evolution of sexually selected traits.

Female monopolization and investment in male sexual traits. We tested whether the variable interspecific relationships observed between pre- and postcopulatory traits are influenced by the degree to which competition allows males to monopolize access to females, our proxy for Parker et al.'s ${ }^{13}$ mate competition loading $a$. To do this, we combined the data presented in Table 1 with additional comparative data sets from the literature, including data on pinnipeds ${ }^{20}$, acanthocephalan parasitic worms $^{19}$ and bushcrickets ${ }^{22}$ (no additional datasets could be located to expand the results of Table 2). On the basis of Parker et al.'s ${ }^{13}$ predictions, we assumed that, across taxa, $a$ would be an increasing function of the proportion of species in each taxonomic group with female monopolization, and that high female monopolization should be associated with increased investment in weaponry and reduced investment in testes size. For each of the taxonomic groups, we thus characterized mating behaviours and territoriality for as many of the species examined in phylogenetic regressions as possible, and considered female monopolization to be present if male-male contest competition influences reproductive success. Specifically, we considered female monopolization to be present in species with female defence polygyny (for example, haremic species), while classifying species with scramble-competition polygyny, resource-defence polygyny, lek polygyny or monogamy to exhibit low or no female monopolization (see Table 3 and Supplementary Note 1). We used this approach to determine taxon-specific proportions of species that exhibit female monopolization (see ref. 31 for a similar classification across taxa) and found that the proportion of female monopolization varied widely among the taxonomic groups examined (Table 3 ). We then converted the effect sizes (partial correlation coefficients $r$ from the multiple regressions

Table 3 | Female monopolization and the relationship between pre- and postcopulatory sexual traits.

ID Taxa

Sexual size dimorphism

Male weapon size

Monopolization classification ${ }^{\star}$

N Weighted $Z_{r}$ Prop. monopol.

1 Primates

$59(67)$

$-1.92$

0.203

0.472

Ungulates $53(59)$

$-0.50$

0.400

Pheasants and allies

35

11.69

0.000

Stalk-eyed flies

26.44

8 (11)

Schistosomes

31 (43)

1.62

0.500

Pinnipeds

11.94

0.097

13

$-10.99$

8 Acanthocephala

$-26.68$

0.640

$\begin{array}{lll}112 & -26.68 & 1.000\end{array}$

9 Bushcrickets

10 Dung beetles

$19 \quad 14.52$

0.000

\begin{tabular}{cr} 
& Male \\
\hline $\boldsymbol{N}$ & Weight \\
\hline $52(54)$ & 2.0 \\
$55(64)$ & 0.18 \\
40 & 0.7 \\
- & - \\
$8(11)$ & 1.0 \\
- & - \\
- & - \\
- & - \\
- & - \\
22 & 6.7
\end{tabular}

$\begin{array}{lll}- & - & 22\end{array}$

Prop.

p. monopol.

$0.231 \quad$ Mating system ${ }^{\dagger}$

0.463 Degree of territorial female defence

0.350

Mating system $\$$

- Degree of territorial female defencell

$\mathrm{N}$, number of species for which sufficient data were available to calculate the proportion of female monopolization from mating or territorial behaviour (the total number of species analysed in multiple regressions are indicated in parentheses); Weighted $Z_{n}$ effect size (that is, partial correlation coefficient $r$ transformed using Fisher's transformation and weighted for sample size); Prop. monopol.; proportion of species within each taxon where males monopolize females.

${ }^{\star}$ The binary classification of female monopolization differed among species based on the best available taxon-specific metric as follows.

†species with monogamous and multi-male mating systems were classified as low monopolization while single-male mating systems were classified as high monopolization.

¥Species with territory defence were classified as low monopolization while species with non-territorial female defence were classified as high monopolization.

\$species with monogamous/non-haremic mating systems were classified as low monopolization while species with polygamous/haremic mating systems were classified as high monopolization.

IAlthough minnows have both group spawning and pair spawning mating behaviours, all species were classified as having low levels of female monopolization due to the external nature of fertilizations.

"Species that do not form mating aggregations were classified as low monopolization while species with mating aggregations were classified as high monopolization.

\#Although dung beetles vary in the degree of sneaking behaviour, females routinely store sperm from different males and these have equal chances of fertilization, thus indicating at best minimal ability of males to monopolize females.

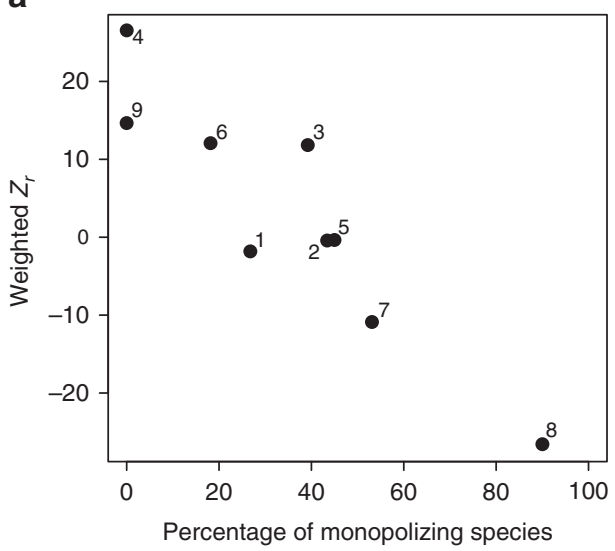

b

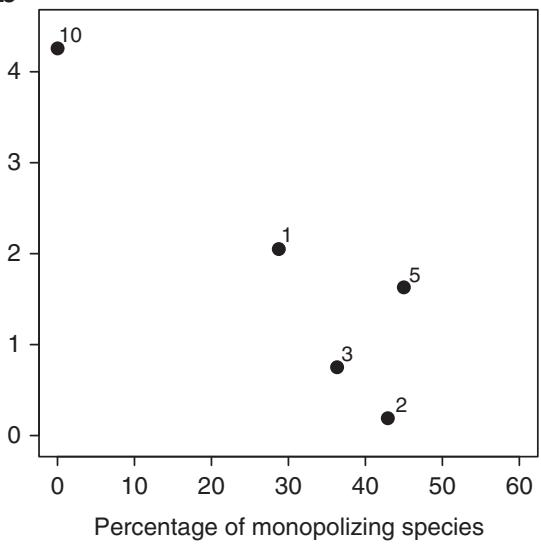

Figure 1 | Covariation between sexual traits relative to the prevalence of female monopolization. Negative Pearson's product moment correlation of the taxon-specific weighted effect sizes $Z_{r}$ of relationships between testes size and (a) SSD or (b) male weapon size, respectively, against the taxon-specific percentages of species exhibiting female monopolization ( (a) Pearson's product moment correlation, $r=-0.92, N=9, P=0.0008$; (b) Pearson's product moment correlation, $r=-0.91, N=5, P=0.05$ ). All values of female monopolization are arcsine square-root transformed and then converted to percentages by multiplying by $180 / \pi$. 
between weapons and testes size), which provide a measure of the strength and direction of the association between pre- and postcopulatory traits, to $Z_{r}$ values using Fisher's transformation weighted by sample size ${ }^{32}$.

In a Pearson's product moment correlation examining the relationship between weighted effect size values and the proportion of female monopolization (arcsine square-root transformed) across nine taxa, $Z_{r}$ was negatively associated with the taxon-specific percentages of species exhibiting female monopolization (Pearson's product moment correlation, $r=-0.92, N=9, P=0.0008$; Fig. 1a). This negative relationship also remained significant when taxonomic groups with either no monopolization ( $0 \%$; Pearson's product moment correlation, $r=-0.89, N=7, P=0.008)$ or complete monopolization $(100 \%$; Pearson's product moment correlation, $r=-0.84, N=8$, $P=0.01)$ were removed from the analysis. Examining the five vertebrate and four invertebrate taxa separately also yielded negative relationships in both cases, although the association among vertebrates was not statistically significant (vertebrates: Pearson's product moment correlation, $r=-0.80, N=5, P=0.11$; invertebrates: Pearson's product moment correlation, $r=-0.99$, $N=4, P=0.04)$. Overall, and in accordance with Parker et al.'s ${ }^{13}$ prediction, these results indicate that negative relationships between pre- and postcopulatory traits are more likely in taxonomic groups where female monopolization is common, while positive relationships between pre- and postcopulatory traits occur in groups where female monopolization is either rare or absent.

We found similar trends when examining the expression of male weapons instead of sexual dimorphism in body size. Although none of the relationships between pre- and postcopulatory sexual traits were significant among the five taxonomic groups with complete data (Table 2), their weighted $Z_{r}$ values tended to decline with an increasing proportion of species classified as monopolizing females (Pearson's product moment correlation, $r=-0.91, N=5, P=0.05$; Fig. $1 \mathrm{~b})$. This result is consistent with the patterns found for SSD, but since the relationship appears to be primarily driven by the dung beetles (data point 10) in our small data set, additional data are clearly needed for a more robust analysis before we can draw more general conclusions.

\section{Discussion}

Our results revealed a potentially unifying pattern of relative resource allocation among sexual traits, at least for traits directly involved in pre- and postcopulatory male-male competition. We found that, although the interspecific relationships between preand postcopulatory sexual characters appear to be inconsistent among taxonomic groups, the strength and direction of these associations are inversely related to the proportion of species exhibiting female monopolization within each group. These results provide empirical support for Parker et al.'s ${ }^{13}$ theoretical prediction that the importance of female monopolization within a taxonomic group should influence the covariance between sexual traits important in pre- and postcopulatory sexual selection.

We found that in taxonomic groups where male contest competition has a strong impact on mate monopolization (for example, pinnipeds), testes size is inversely related to sexual size dimorphism, a premating sexual trait involved in determining access to females. By contrast, these interspecific relationships were positive in taxa where female monopolization is rare or absent. Although limited to only five taxonomic groups and thus with relatively low statistical power, we found a similar pattern for external structures known to be involved in male-male contest competition, with the strength of the interspecific weapon size-testes size relationships tending to decline with increasing percent female monopolization across taxa. Compared with SSD, however, none of the intra-taxonomic relationships were statistically significant. While this may seem somewhat surprising given the assumed expense of these weapons and their importance in mediating postcopulatory sexual selection through female monopolization (see below), it should be noted that all taxa included in this analysis exhibited less than 50\% female monopolization. Consequently, it is too early to draw firm conclusions, but the overall similarity to our findings for SSD suggests that the dependence of covariance between sexual traits on female monopolization may be a more general pattern. We thus encourage further intraspecific and/or comparative studies on these links for a broader empirical examination of Parker et al.' $\mathrm{s}^{13}$ theoretical predictions.

Overall, our data suggest that if male-male contest competition determines access to mates, testes size might be traded against investment in precopulatory sexual traits. Alternatively, postcopulatory sexual selection might simply become increasingly less important as the level of female monopolization increases, being negligible where dominant males can prevent any other male from mating with the females in their harems (that is, complete female monopolization). Therefore, testes size (and possibly other postcopulatory sexual traits) might evolve as a direct function of the intensity of premating sexual selection through male-male contest competition. In fact, similar to our inter-taxonomic comparison, closer examination of the pinnipeds reveals that a negative relationship between testes size and SSD is found among haremic but not among non-haremic species ${ }^{20}$. This result corroborates the findings of the present study within a single taxonomic group by also suggesting that negative covariance between pre- and postcopulatory male investments may be limited to groups of species where male mating success is associated with female monopolization.

In other taxa, where female monopolization is less common, mate searching or postcopulatory sexual selection might be more important. However, even if sperm competition is the primary target of sexual selection within a taxonomic group, species exhibiting intense sperm competition are more likely to also show some level of precopulatory male competition (through males' attempts to avoid sperm competition) than species where selection on male-male competition in general is less intense, which could then result in positive interspecific covariation between pre- and postcopulatory sexual traits. Male-male competition may explain a smaller proportion of the variance in male mating success either because male control over female mating is constrained (for example, external fertilization in minnows) or because sexual selection acts on traits involved in male-male contests as well as traits important for female mate choice (for example, song in bushcrickets ${ }^{33}$ or plumage and other sexual traits in pheasants and allies ${ }^{34}$ ). These morphological or physiological male 'ornaments' can also incur considerable energetic costs (for example, bushcricket song ${ }^{33}$ ). By diverting resource allocation away from male contest traits, both pre- and postcopulatory sexual traits associated with male-male competition might be under positive selection (either by concerted or independent evolution), and negative covariance might be found between these traits combined and the expression of male ornaments rather than between the competition traits themselves. These patterns are comparable to previous reports documenting that strong selection for sexually selected traits can generate positive covariance among pre- and postcopulatory traits that combined are negatively related to life-history traits such as somatic maintenance, immunity or longevity ${ }^{35,36}$. There is some, albeit indirect, evidence supporting the above hypothesis that competition traits combined might be inversely related to 
ornaments. A recent study on the macroevolutionary gains and losses of various sexual characters among pheasant species suggests that characters associated with male-male contests tend to show a greater rate of gains than losses, whereas intersexual display traits show the opposite trend ${ }^{34}$. Whether these opposite evolutionary trajectories are the result of trade-offs as opposed to independent evolution and whether similar trends exist in other taxa remains to be seen.

Our finding of a dependence of pre- and postcopulatory trait covariance on female monopolization may also extend predictions resulting from resource acquisition-allocation models. These models predict that the sign and strength of between-trait covariance are the result of differences in the mean and variance of resource acquisition relative to resource allocation ${ }^{37}$. Specifically, if males are better at acquiring or defending resources and use them more efficiently, they can afford to invest relatively more in both pre- and postcopulatory reproductive traits than others, resulting in positive trait covariation across males even in the presence of intra-male allocation trade-offs ${ }^{38}$. A good example is presented by stalk-eyed flies, in which the titre of juvenile hormone $(\mathrm{JH})$, a critical regulator of development and metamorphosis in insects, mediates an allocation trade-off between the sexually selected eye stalks and testes within developing males ${ }^{39}$. Despite this intra-male trade-off, the two traits covary positively across males in wild populations ${ }^{40}$, possibly through pleiotropic effects of $\mathrm{JH}$ or variation in larval resource acquisition combined with a nutrition-dependent $\mathrm{JH}$ titre ${ }^{41,42}$. By contrast, a negative association between sexual traits would be expected if males vary little in the total acquired resources but greatly in their allocation to different traits ${ }^{38}$.

Although the interaction between resource acquisition and allocation prevents direct inferences about trait covariance at the macroevolutionary scale based on such intraspecific patterns ${ }^{38}$, the general arguments about resource-dependent trait covariance should also apply to higher taxonomic levels ${ }^{43}$. For example, populations or species may differ in their diet type and/or quality, in the abundance and distribution of resources, or in life-history investments (for example, metabolism or survival). These effects may ultimately determine the relative allocation to sexual traits and the potential for selection to act on them ${ }^{13,43}$. Whichever the taxonomic level, any biases in resource acquisition and allocation to pre- and postcopulatory male-male competition may not be independent of the level of female monopolization. First, resource allocation may determine the expression of precopulatory contest traits, thereby influencing a male's ability to prevent rival males from mating with his monopolized females and ultimately determining the degree of postcopulatory sexual selection experienced (and selection on the testes). Second, depending on the importance of female monopolization in explaining male fitness and on which trait has the greatest fitness gain with any marginal increase in size, the acquired resources might be channelled preferentially to either pre- or postcopulatory sexual traits, respectively (see above). Finally, female monopolization might influence resource acquisition itself, with the defence of harems limiting the opportunities to forage and acquire resources for (future) allocation to both pre- and postcopulatory sexual traits. However, to specifically examine such links between resource acquisition-allocation and female monopolization as factors mediating coevolution among sexual traits, more detailed information on the female monopolization capacity than our binary coding of presence/absence would be necessary (for example, mean or maximum harem size and its variance and distribution among species).

Although our comparative study has revealed potentially unifying macroevolutionary patterns, detailed intraspecific analyses are needed to understand the basis and adaptive significance of the general evolutionary trends established by such comparative studies (for example, ref. 44). Intraspecific analyses are particularly important for discerning relationships among traits due to differences in resource allocation (for example, allocation trade-offs) from other potential sources of covariation among traits (for example, genetic effects, independent selection). To our knowledge, intraspecific data on covariation among pre- and postcopulatory investments relative to female monopolization are currently lacking. It would be interesting to see, however, whether the direction and strength of the intra-population relationship between pre- and postcopulatory traits varies across populations that differ naturally or through experimental manipulation in the intensity of male contest competition.

For the purposes of our study, we used relative testes size as a proxy for male investment in sperm production. It should be noted, however, that adaptations to sperm production in response to postcopulatory sexual selection can also involve increases in the density and functional efficiency of the sperm-producing tissue across testes of identical size ${ }^{45-48}$. Moreover, in addition to sperm competition, selection on testes size can occur through selection against sperm depletion ${ }^{49}$, with males that monopolize more females and thus mate more frequently losing paternity due to sperm depletion ${ }^{50}$. Thus, although the target of selection may be the competitiveness of future ejaculates rather than that of the current ejaculate, similar patterns of investment in sperm production would be expected in both scenarios. Finally, relative testes size can be more intimately linked to premating sexual selection, in that, at least in vertebrates, testes also produce testosterone that can be associated with male aggression and mating success ${ }^{51}$. As a result, testes can be enlarged to primarily increase the output of testosterone, potentially at the expense of considerably less sperm-producing tissue ${ }^{52,53}$. Although these different types of selection on testes are unlikely to have any appreciable effect on our key results given the scope of our study, it is important to be aware of such sources of potential unexplained variation when examining the covariance between pre- and postcopulatory sexual traits.

In conclusion, we demonstrate that interspecific relationships between pre- and postcopulatory traits are positive in taxonomic groups with rare or no female monopolization but gradually approach zero and ultimately become negative as female monopolization becomes more prevalent within taxonomic groups. These results indicate that intra-taxonomic variance in the strength of female monopolization influences the covariance between sexual traits and reveal important information on how variance in allocation strategies differentially influences the evolution of sexual traits.

\section{Methods}

Data collection and study taxa. We collected data on premating weapons, male and female body size, and testes mass or size from the literature for primates, ungulates, pheasants and allies, minnows, stalk-eyed flies, dung beetles and schistosomes (Supplementary Tables 1-6; for data of the minnows exactly as used in our analyses, see ref. 27). These taxa exhibit broad variance in the levels of multiple mating, mating systems and mating strategies (Supplementary Note 1). We were not able to account for every taxon-specific difference characterizing the strength of sexual selection, but we considered the variance in the strength of sexual selection within taxa to be more important for the purposes of our study than absolute differences in selection intensity.

We focused our analyses on traits that influence male fitness based on intrasexual contests, such as male armaments or sexual size dimorphism, rather than including traits used in female mate choice, for two reasons. First, traits used in female mate choice are extremely diverse, including acoustic, visual, olfactory or behavioural display traits, thereby considerably complicating comparisons across different taxa. In contrast, the diversity in weaponry associated with male contest competition is more readily comparable in terms of resource allocation. Second, studies of the evolution of sexually selected traits tend to be strongly biased towards 
ornaments and female mate choice, with comparative studies on the evolution of weapons lagging far behind ${ }^{54}$

To calculate sexual size dimorphism (SSD = male size/female size; for example, ref. 55), we used body size measured as body mass in all vertebrate taxa except minnows (standard length) and a measure of body length that is widely used for each of the invertebrate taxa except dung beetles (body mass). The use of mass and linear size indices had no effect on the main results (Supplementary Note 2). Note, however, that to avoid the problematic use of ratios in phylogenetic models, we calculated SSD as $\log$ (male size/female size), which is equivalent to $\log ($ male size $-\log ($ female size). SSD can be biased towards males or females, due to three main processes. First, while selection may favour small males in aerial competition due to higher maneuverability ${ }^{56,57}$, the more common contests on the ground typically select for larger males ${ }^{57-60}$. Second, female-biased SSD can arise through selection on female fecundity, possibly enhanced by male preference for large females ${ }^{61,62}$. Third, natural selection may favour different body sizes if dimorphic species can avoid resource competition or increase feeding efficiency by exploiting a wider range of resources compared with sexually monomorphic species ${ }^{63-65}$. In our study, we focused on taxa in which sexual selection is thought to play a critical role in the evolution of SSD in that relatively large males are known to have a mating advantage, and sexual selection is thus likely to act on male size independent of that on female size. This does not entirely reject the possibility that some level of the variation in SSD can be attributed to fecundity selection in females (particularly among insects). Yet, such fecundity selection should not alter our main analysis across taxonomic groups (Fig. 1). First, in the case of fecundity selection, females tend to be larger than males (that is, reversed SSD), but, as sexual selection on male size becomes more intense, the difference between male and female size should decrease. Across species, this would still result in a positive relationship between male/female SSD and the intensity of sexual selection on males, and it is such variance that our analyses captured. Second, we hypothesized that conducting parallel analyses between SSD and weapon size or between vertebrates and invertebrates (that is, where fecundity selection is less or more important, respectively) should control for such confounding effects.

Relationships between pre- and postcopulatory sexual traits. To assess covariance between pre- and postcopulatory sexual traits while accounting for statistical non-independence of data points by shared ancestry of species, we conducted multiple regressions using phylogenetic generalized least-squared (PGLS) regressions $^{66}$, based on published molecular phylogenies for each taxon (Supplementary Figs 1-6, for minnows see ref. 27). Using maximum-likelihood methods, these models estimate the phylogenetic scaling parameter $\lambda$ to evaluate the phylogenetic relationship of the covariance in the residuals ${ }^{66}$. We used likelihood ratio tests to establish whether the models with the maximum-likelihood value of $\lambda$ differed from models with values of $\lambda=0$ or $\lambda=1$, respectively, with $\lambda$ not significantly different from 0 indicating phylogenetic independence and $\lambda$ not significantly different from 1 indicating a complete phylogenetic association of the traits ${ }^{66}$. Except for the primate phylogeny, branch lengths were unknown. For consistency, we thus assigned equal branch lengths to all phylogenies.

In all analyses testing for relationships between pre- and postcopulatory traits, we used testes size as the dependent variable and the precopulatory sexual trait (for example, sexual size dimorphism) as the independent variable and accounted for allometric effects by including male body size as a covariate ${ }^{67,68}$. All analyses were performed using the statistical package R version 2.15.2 (ref. 69), and all nonnormal data distributions were transformed by logarithmic transformations (in the case of pheasant spur length as $Y^{\prime}=\log (Y+0.001)$ due to zero values) to meet the parametric requirements of the statistical models.

Female monopolization and investment in male sexual traits. To test Parker et al.' $\mathrm{s}^{13}$ predictions on the covariation between investments in pre- and postcopulatory reproductive traits relative to the degree to which competition allows males to monopolize access to females (the mate-competition loading, $a$ ), we evaluated the relationship between the sample-size weighted effect size $(r)$ of partial correlation coefficients between pre- and postcopulatory sexual traits derived from our multiple regression models (data taken from Table 1 and published studies ${ }^{19,20,22}$ or from Table 2, respectively) and the proportion of species within each taxon that exhibit female monopolization (Table 3). Because sample sizes differed among the taxa examined, we converted the $r$-values listed in Tables 1 or 2 and calculated from published studies to $Z_{r}$-values using Fisher's transformation and weighted them by sample size to obtain a weighted $Z_{r}$ for each $\operatorname{taxon}^{32}$. To determine the proportion of species within each taxon that exhibited female monopolization, we characterized mating behaviours and territoriality for as many of the species examined in phylogenetic regressions as possible. Specifically, we focused on categorizing the number of species in which male-male contest competition influences reproductive success within each taxon. These classifications (described in detail in the Supplementary Note 1) were applied to all nine taxa for which data on SSD as the premating trait involved in male-male competition were available. We used the proportion of female monopolization in each taxon as a proxy measure for the mate-competition loading factor, $a$ (as defined by ref. 13). We examined the relationship between $Z_{r}$ and female monopolization using a linear regression on arcsine square-root transformed monopolization values. Although criticized by Warton and $\mathrm{Huil}^{70}$, this transformation was equally successful in improving the residual distribution as the recommended empirical logistic transformation, but it retained a directly interpretable $x$-axis (that is, $0-100 \%$ monopolization), which we rated more important than the slope parameters. Regardless of the transformation (and even without transformation), the effect size of the inter-taxonomic relationship remained identical. Because of the small sample sizes in these analyses, we estimated the $P$-values using randomization tests (R package $l m P e r m)^{69}$ with 1,000 permutations.

\section{References}

1. Darwin, C. The Descent of Man and Selection in Relation to Sex (John Murray, London, UK, 1871).

2. Andersson, M. Sexual Selection (Princeton University Press, Princeton, NJ, USA, 1994).

3. Jennions, M. D. \& Petrie, M. Why do females mate multiply? A review of the genetic benefits. Biol. Rev. 75, 21-64 (2000).

4. Parker, G. A. Sperm competition and its evolutionary consequences in the insects. Biol. Rev. 45, 526-567 (1970).

5. Eberhard, W. G. Female Control: Sexual Selection by Cryptic Female Choice (Princeton University Press, Princeton, NJ, USA, 1996).

6. Thornhill, R. Cryptic female choice and its implications in the scorpionfly Harpobittacus nigriceps. Am. Nat. 122, 765-788 (1983).

7. Andersson, M. \& Simmons, L. W. Sexual selection and mate choice. Trends Ecol. Evol. 21, 296-302 (2006).

8. Kvarnemo, C. \& Simmons, L. W. Polyandry as a mediator of sexual selection before and after mating. Philos. Trans. R. Soc. Lond. B 368, 20120042 (2013).

9. Dewsbury, D. A. Ejaculate cost and male choice. Am. Nat. 119, 601-610 (1982).

10. Olsson, M., Madsen, T. \& Shine, R. Is sperm really so cheap? Costs of reproduction in male adders, Vipera berus. Proc. R. Soc. Lond. B 264, 455-459 (1997).

11. Emlen, D. J. Costs and the diversification of exaggerated animal structures. Science 291, 1534-1536 (2001).

12. Allen, B. J. \& Levinton, J. S. Costs of bearing a sexually selected ornamental weapon in a fiddler crab. Funct. Ecol. 21, 154-161 (2007).

13. Parker, G. A., Lessells, C. M. \& Simmons, L. W. Sperm competition games: a general model for pre-copulatory male-male competition. Evolution 67, 95-109 (2013).

14. Simmons, L. W. \& Emlen, S. T. Evolutionary trade-off between weapons and testes. Proc. Natl Acad. Sci. USA 103, 16346-16351 (2006).

15. Puniamoorthy, N., Blanckenhorn, W. U. \& Schäfer, M. A. Differential investment in pre- vs. post-copulatory sexual selection reinforces a crosscontinental reversal of sexual size dimorphism in Sepsis punctum (Diptera: Sepsidae). J. Evol. Biol. 25, 2253-2263 (2012).

16. Galeotti, P. et al. Sperm traits negatively covary with size and asymmetry of a secondary sexual trait in a freshwater crayfish. PLoS One 7, e43771 (2012).

17. Malo, A. F., Roldan, E. R. S., Garde, J., Soler, A. J. \& Gomendio, M. Antlers honestly advertise sperm production and quality. Proc. R. Soc. Lond. B 272, 149-157 (2005).

18. Kelly, C. D. Sperm investment in relation to weapon size in a male trimorphic insect? Behav. Ecol. 19, 1018-1024 (2008).

19. Poulin, R. \& Morand, S. Testes size, body size and male-male competition in acanthocephalan parasites. J. Zool. 250, 551-558 (2000).

20. Fitzpatrick, J. L., Almbro, M., Gonzalez-Voyer, A., Kolm, N. \& Simmons, L. W. Male contest competition and the coevolution of weaponry and testes in pinnipeds. Evolution 66, 3595-3604 (2012).

21. Gage, M. J. G., Parker, G. A., Nylin, S. \& Wiklund, C. Sexual selection and speciation in mammals, butterflies and spiders. Proc. R. Soc. Lond. B 269, 2309-2316 (2002).

22. Wedell, N. Spermatophore size in bushcrickets: comparative evidence for nuptial gifts as a sperm. Evolution 47, 1203-1212 (1993).

23. Harcourt, A. H., Harvey, P. H., Larsen, S. G. \& Short, R. V. Testis size, body weight and breeding system in primates. Nature 293, 55-57 (1981).

24. Soulsbury, C. D. Genetic patterns of paternity and testes size in mammals. PLoS One 5, e9581 (2010).

25. Birkhead, T. R. \& Møller, A. P. Sperm Competition and Sexual Selection (Academic Press, San Diego, CA, USA, 1998).

26. Simmons, L. W. \& Fitzpatrick, J. L. Sperm wars and the evolution of male fertility. Reproduction 144, 519-534 (2012).

27. Pyron, M., Pitcher, T. E. \& Jacquemin, S. J. Evolution of mating systems and sexual size dimorphism in North American cyprinids. Behav. Ecol. Sociobiol. 67, 747-756 (2013).

28. Clutton-Brock, T. H., Harvey, P. H. \& Rudder, B. Sexual dimorphism, socionomic sex ratio and body weight in primates. Nature 269, 797-800 (1977).

29. Webster, M. S. Sexual dimorphism, mating system and body size in New World blackbirds (Icterinae). Evolution 46, 1621-1641 (1992).

30. Gonzalez-Voyer, A., Fitzpatrick, J. L. \& Kolm, N. Sexual selection determines parental care patterns in cichlid fishes. Evolution 62, 2015-2026 (2008). 
31. Shultz, S. \& Dunbar, R. Encephalization is not a universal macroevolutionary phenomenon in mammals but is associated with sociality. Proc. Natl Acad. Sci. USA 107, 21582-21586 (2010).

32. Rosenthal, R. Meta-Analytic Procedures for Social Research (Sage, London, UK, 1991).

33. Gwynne, D. T. Katydids and Bush-Crickets: Reproductive Behavior and Evolution of the Tettigoniidae (Cornell University Press, Ithaca, 2001).

34. Kimball, R. T., St. Mary, C. M. \& Braun, E. L. A macroevolutionary perspective on multiple sexual traits in the Phasianidae (Galliformes). Int. J. Evol. Biol. 2011, 423938 (2011).

35. Simmons, L. W. \& Roberts, B. Bacterial immunity traded for sperm viability in male crickets. Science 309, 2031 (2005).

36. Kirkwood, T. B. L. \& Austad, S. N. Why do we age? Nature 408, 233-238 (2000).

37. Roff, D. A. \& Fairbairn, D. J. The evolution of trade-offs: where are we? J. Evol. Biol. 20, 433-447 (2007).

38. van Noordwijk, A. J. \& de Jong, G. Acquisition and allocation of resources: their influence on variation in life history tactics. Am. Nat. 128, 137-142 (1986).

39. Fry, C. L. Juvenile hormone mediates a trade-off between primary and secondary sexual traits in stalk-eyed flies. Evol. Dev. 8, 191-201 (2006).

40. Cotton, S., Small, J., Hashim, R. \& Pomiankowski, A. Eyespan reflects reproductive quality in wild stalk-eyed flies. Evol. Ecol. 24, 83-95 (2010).

41. Osorio, S., Piulachs, M. D. \& Bellés, X. Feeding and activation of corpora allata in the cockroach Blattella germanica (L.) (Dictyoptera, Blattellidae). J. Insect Physiol. 44, 34-38 (1998).

42. Trumbo, S. T. \& Robinson, G. E. Nutrition, hormones and life history in burying beetles. J. Insect Physiol. 50, 383-391 (2004).

43. Shutler, D. Sexual selection: when to expect trade-offs. Biol. Lett. 7, 101-104 (2011).

44. Leroi, A. M., Rose, M. R. \& Lauder, G. V. What does the comparative method reveal about adaptation? Am. Nat. 143, 381-402 (1994).

45. Lüpold, S., Linz, G. M., Rivers, J. W., Westneat, D. F. \& Birkhead, T. R. Sperm competition selects beyond relative testes size in birds. Evolution 63, 391-402 (2009).

46. Lüpold, S., Wistuba, J., Damm, O. S., Rivers, J. W. \& Birkhead, T. R. Sperm competition leads to functional adaptations in avian testes to maximize sperm quantity and quality. Reproduction 141, 595-605 (2011).

47. Ramm, S. A. \& Stockley, P. Sperm competition and sperm length influence the rate of mammalian spermatogenesis. Biol. Lett. 6, 219-221 (2010).

48. Schärer, L. \& Vizoso, D. B. Phenotypic plasticity in sperm production rate: there's more to it than testis size. Evol. Ecol. 21, 295-306 (2007).

49. Vahed, K. \& Parker, D. J. The evolution of large testes: Sperm competition or male mating rate? Ethology 118, 107-117 (2012).

50. Preston, B. T., Stevenson, I. R., Pemberton, J. M. \& Wilson, K. Dominant rams lose out by sperm depletion: a waning success in siring counters a ram's high score in competition for ewes. Nature 409, 681-682 (2001).

51. Wingfield, J. C., Hegner, R. E., Dufty, Jr A. M. \& Ball, G. F. The 'challenge hypothesis': Theoretical implications for patterns of testosterone secretion, mating systems, and breeding strategies. Am. Nat. 136, 829-846 (1990).

52. Costa, D. S., Paula, T. A. R. \& Matta, S. L. P. The intertubular compartment morphometry in capybaras (Hydrochoerus hydrochaeris) testis. Anim. Reprod. Sci. 91, 173-179 (2006).

53. Herrera, E. A. \& Macdonald, D. W. Aggression, dominance, and mating success among capybara males (Hydrochaeris hydrochaeris). Behav. Ecol. 4, 114-119 (1993).

54. Emlen, D. J. The evolution of animal weapons. Annu. Rev. Ecol. Syst. 39, 387-413 (2008)

55. Fairbairn, D. J. in Sex, Size and Gender Roles. (eds Blanckenhorn, W. U., Fairbairn, D. J. \& Székely, T.) 1-10 (Oxford University Press, Oxford, UK, 2007).
56. Andersson, M. \& Norberg, R. A. Evolution of reversed sexual size dimorphism and role partitioning among predatory birds, with a size scaling of flight performance. Biol. J. Linn. Soc. 15, 105-130 (1981)

57. Székely, T., Reynolds, J. D. \& Figuerola, J. Sexual size dimorphism in shorebirds, gulls, and alcids: the influence of sexual and natural selection. Evolution 54, 1404-1413 (2000).

58. Clutton-Brock, T. H., Guinness, F. E. \& Albon, S. D. Red Deer: Behaviour and Ecology of Two Sexes (Edinburgh University Press, Edinburgh, 1985).

59. Lindenfors, P. Sexually antagonistic selection on primate size. J. Evol. Biol. 15, 595-607 (2002).

60. Lindenfors, P., Tullberg, B. S. \& Biuw, M. Phylogenetic analyses of sexual selection and sexual size dimorphism in pinnipeds. Behav. Ecol. Sociobiol. 52, 188-193 (2002)

61. Shine, R. Sexual selection and size dimorphism in the Amphibia. Copeia 1979, 297-306 (1979).

62. Prenter, J., Elwood, R. W. \& Montgomery, W. I. Sexual size dimorphism and reproductive investment by female spiders: a comparative analysis. Evolution 53, 1987-1994 (1999).

63. Selander, R. K. Sexual dimorphism and differential niche utilization in birds. Condor 68, 113-151 (1966)

64. Shine, R. Ecological causes for the evolution of sexual dimorphism: a review of the evidence. Q. Rev. Biol. 64, 419-461 (1989).

65. Thom, M. D., Harrington, L. A. \& Macdonald, D. W. Why are American mink sexually dimorphic? A role for niche separation. Oikos 105, 525-535 (2004).

66. Freckleton, R. P., Harvey, P. H. \& Pagel, M. Phylogenetic analysis and comparative data: a test and review of evidence. Am. Nat. 160, 712-726 (2002).

67. Freckleton, R. P. On the misuse of residuals in ecology: regression of residuals vs. multiple regression. J. Anim. Ecol. 71, 542-545 (2002).

68. García-Berthou, E. On the misuse of residuals in ecology: testing regression residuals vs. the analysis of covariance. J. Anim. Ecol. 70, 708-711 (2001)

69. R Development Core Team. R: A language and environment for statistical computing (R Foundation for Statistical Computing, Vienna, Austria, 2012).

70. Warton, D. I. \& Hui, F. K. C. The arcsine is asinine: the analysis of proportions in ecology. Ecology 92, 3-10 (2011).

\section{Acknowledgements}

We thank G. A. Parker and R. D. Montgomerie for valuable comments on our manuscript, S. Shultz for helpful discussions, and the Swiss National Science Foundation (fellowship PA00P3_134191 to S.L.), National Science Foundation (grant DEB-1145965 to S.L.) and Australian Research Council (Discovery Project DP130100618 to L.W.S. Future Fellowship FT110100500 to J.L.T.; Australian Postdoctoral Fellowship Discovery Project DP1096253 to J.L.F.) for financial support.

\section{Author contributions}

All authors designed the study and collected the data; S.L. analysed the data; S.L. and J.L.F. wrote the manuscript and all authors edited its final version.

\section{Additional information}

Supplementary Information accompanies this paper at http://www.nature.com/ naturecommunications

Competing financial interests: The authors declare no competing financial interests.

Reprints and permission information is available online at http://npg.nature.com/ reprintsandpermissions/

How to cite this article: Lüpold, S. et al. Female monopolization mediates the relationship between pre- and postcopulatory sexual traits. Nat. Commun. 5:3184 doi: 10.1038/ncomms4184 (2014). 